\title{
Time-Dependence of the Displacement Adsorption Behavior of Poly(methyl methacrylate) and Polystyrene System at the Solid-Liquid Interface as Studied by ESR
}

\author{
Kazutoshi Kobayashi, ${ }^{\dagger}$ Atsuko Dochi, Hirofumi Yajima, \\ and Ryuichi ENDO \\ Department of Applied Chemistry, Faculty of Science, Science University of Tokyo, \\ Kagurazaka, Shinjuku-ku, Tokyo 162, Japan
}

(Received February 2, 1993)

\begin{abstract}
The displacement adsorption behavior of poly(methyl methacrylate) (PMMA) and polystyrene (PS) system onto a nonporous silica surface from solution was studied by utilizing the ESR method. The fractions of train segments for these polymers were determined from the ESR spectra analyses, and these values were compared with those of the individual adsorption. When PS was preadsorbed on silica surface to saturation and then excess PMMA solution of known concentration was added and data taken as a function of adsorption time, the amount of PS desorbed from silica surface was found to increase proportionally with adsorption time until finally PS was completely replaced by PMMA. With the displacement of PS from the silica surface, the amount of adsorption for PMMA which saturates the silica surface is obviously lower than that when adsorbed on silica surface individually; that is, the adsorbed PMMA has a flatter conformation than that in the individual adsorption. It also takes a long time for PMMA to adsorb on the silica surface compared with the individual adsorption, because a greater number of PS-silica surface attachments must be ruptured in order to effect displacement.
\end{abstract}

KEY WORDS Displacement Adsorption / ESR / Polystyrene / Poly(methyl methacrylate) / Solid-Liquid Interface

A number of techniques have been applied to the study of the polymers adsorbed at the solid-liquid interface. ${ }^{1-16}$ Among the molecular models proposed for characterizing polymer molecules adsorbed from solution onto a solid surface, a "loop-train-tail" model is now widely accepted. ${ }^{1}$ In order to discuss the conformation of an adsorbed polymer molecule, it is important to estimate the fraction of train segments in all segments of the adsorbed polymer, $p$.

Several studies have been reported on the competitive and displacement adsorption of polymers. ${ }^{8,12,17-28}$ Kawaguchi et al. ${ }^{23}$ studied the competitive and displacement adsorption of PS and poly(ethylene oxide) (PEO) and reported that PEO adsorbs preferentially over PS, and the preferential adsorption of large molecules over small molecules occurs even in the presence of different species. Thies ${ }^{12}$ studied the competitive and displacement adsorption of PS and PMMA mixtures from trichloroethylene solution using IR spectroscopy. He reported the reversibility of adsorption processes in such systems. The present authors ${ }^{8}$ reported the competitive adsorption behavior of PS and PMMA mixtures and discussed the conformation of both adsorbed polymers under this condition. We also discussed the difference in the adsorption ability of styrene and methyl methacrylate on the silica surface.

\footnotetext{
${ }^{\dagger}$ Present address: Frontier Research Program, The Institute of Physical and Chemical Research (RIKEN), Hirosawa 2-1, Wako-shi, Saitama 351-01, Japan.
} 
As reported in our previous paper, ${ }^{5}$ the value of $p$ estimated by the IR method is underestimated compared with that estimated by the ESR method because the IR method evaluates only the segments adsorbed through a hydrogen bond. Therefore, the ESR method is effective for an accurate evaluation of the state of the adsorbed polymer. We have now studied the displacement adsorption behavior of PMMA and PS system using the ESR method, based on estimation of the value of $p$.

Several studies have been reported on the competitive and displacement adsorption of PMMA and PS system. ${ }^{8,12,24,28}$ However, most of them reported only the amounts of adsorption and desorption. The purpose of this study is to utilize the ESR method to explore the displacement adsorption behavior of PMMA and PS system onto a nonporous silica surface from carbon tetrachloride solution. Therefore, we report the conformation of both polymers accompanied by displacement as the principal objective. In this paper, we deal with only the adsorption-time dependence of the displacement adsorption behavior of PMMA solution and silica adsorbents which is preadsorbed by PS. Incidentally, we do not deal with a system which PMMA is preadsorbed on silica surface and then PS solution is added, because PMMA can not be displaced by PS as reported in previous papers. ${ }^{8,12}$

Since it is impossible to deal with a system having both polymer components spin-labeled, or to analyze the ESR spectrum for the dynamic behavior of PMMA and PS individually, we deal with two kinds of displacement adsorption systems: (1) the displacement adsorption of spin-labeled PMMA (SLPMMA) and normal PS (NL-PS), (2) the displacement adsorption of normal PMMA (NL-PMMA) and spin-labeled PS (SL-PS).

\section{EXPERIMENTAL}

\section{Materials}

The polymer samples were basically the same as those described in our previous paper. ${ }^{8}$
Table I. Characterization of the polymer samples used

\begin{tabular}{lcc}
\hline Sample name & $\bar{M}_{w}{ }^{\mathrm{a}}$ & $\bar{M}_{\mathrm{w}} / \bar{M}_{n}{ }^{\mathrm{a}}$ \\
\hline SL-PMMA & 224000 & 1.3 \\
NL-PMMA & 219000 & 1.3 \\
SL-PS & 221000 & 1.5 \\
NL-PS & 207000 & 1.1 \\
\hline
\end{tabular}

a $\bar{M}_{w}$ and $\bar{M}_{w} / \bar{M}_{n}$ were determined by means by GPC in tetrahydrofuran (THF).

NL-PMMA and SL-PMMA were prepared by an anionic polymerization. ${ }^{2}$ NL-PS was purchased from Pressure Chemical Co. SL-PS was prepared from the procedure of Regen. ${ }^{29}$ The polymer samples used in this work are characterized in Table I.

Cabosil M5, with a specific surface area of $220 \mathrm{~m}^{2} \mathrm{~g}^{-1}$ for nitrogen adsorption, was used as an adsorbent. The silica had a $2.2 \mathrm{~nm}^{-2}$ silanol density on its surface. The silica was essentially nonporous and had an average particle size of $14 \mathrm{~nm}$.

Carbon tetrachloride used in this work was spectroscopic grade and was used without further purification.

\section{Techniques of Measurements}

The experimental techniques for the adsorption of polymers on the silica adsorbents were basically the same as those described in previous papers. ${ }^{5-8}$ The polymer solutions for PMMA and PS, respectively, containing initial concentrations of $7 \times 10^{-3} \mathrm{~g} \mathrm{~cm}^{-3}$ and $2 \times$ $10^{-3} \mathrm{~g} \mathrm{~cm}^{-3}$, were added to the silica adsorbents $(0.1 \mathrm{~g})$, and the mixtures were stirred with a magnetic stirrer. The silica dispersion was separated by the use of centrifuge. The amount of adsorption, $A\left(\mathrm{~g} \mathrm{~m}^{-2}\right)$, for PMMA was determined by IR spectroscopy by measuring the difference in the polymer concentration before and after adsorption. The characteristic band at $1732 \mathrm{~cm}^{-1}$ was utilized for IR analysis. The value of $A$ for PS was determined by means of UV spectroscopy by measuring the decrease in the intensity at $262 \mathrm{~nm}$ after the adsorption. 
The structures of the adsorbed polymers were determined from the ESR spectra analyses. ${ }^{1,6}$

The displacement adsorption studies on PMMA-PS system were carried out as follows. In the first place, PS were preadsorbed on silica adsorbents to saturation, and the supernatant solutions were removed. Then excess PMMA solutions of known concentration were added to silica adsorbent. Therein, the concentrations of PMMA and PS solutions and the weight of silica adsorbents used, were the same as with the individual adsorption experiments. The mixtures were stirred for various amounts of adsorption time and separated by the use of centrifuge. The values of $A$ for PMMA, $A_{\text {PMMA }}$, and the values of $A$, for PS, $A_{\mathrm{PS}}$, were measured. The ESR spectra of the adsorbed polymers were also measured.

Analyses of the ESR spectra of the adsorbed spin-labeled polymers were performed using the same procedure described in previous papers. $^{1,6}$

\section{RESULTS AND DISCUSSION}

\section{Adsorption of Individual Polymers}

The amounts of individual adsorption of polymers from carbon tetrachloride solution are plotted against the adsorption time in Figure 1. At least $90 \%$ of the adsorption occurred in less than 2 hours after adding the polymer solution to silica adsorbent, the adsorption being practically complete after 4 hours. As shown in Figure 1, the amounts of saturated adsorption for SL-PMMA and NL-PMMA are almost the same. A similar result was obtained for the adsorption of SL-PS and NL-PS. The influence of the spin-label agent on the amounts of the adsorption is negligible, as the spin-label concentration of the two labeled polymer samples are extremely low. The numbers in the graph are the values of $p$ at that point. The values of $p$ for both polymers decrease gradually with the adsorption time and remained practically constant after 4 hours.

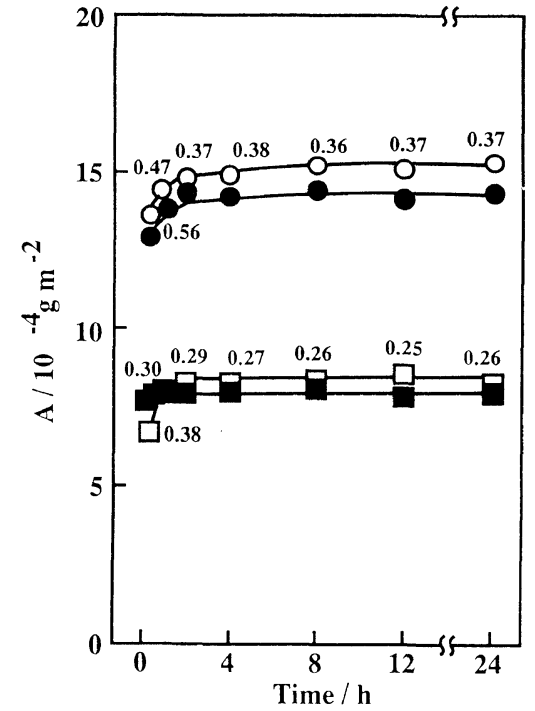

Figure 1. Plots of the adsorbed amounts of polymers against the adsorption time. The numbers in the graph are the values of $p$ at that point. $\bigcirc$, SL-PMMA; $O$, NLPMMA; $\square$, SL-PS; $\square$, NL-PS.

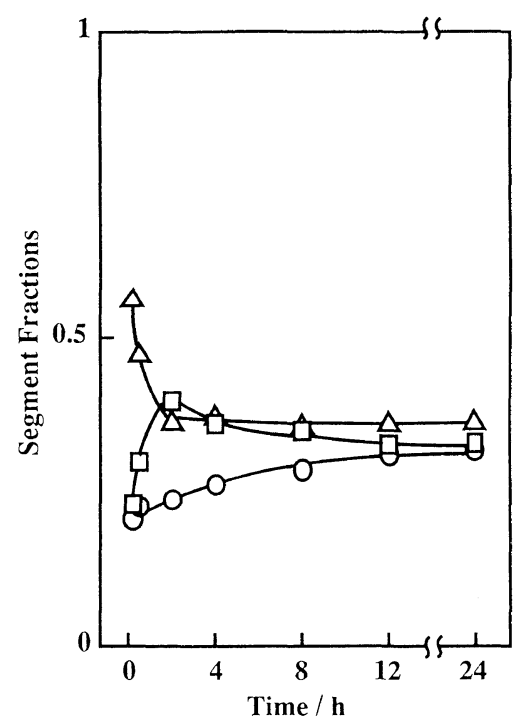

Figure 2. Plots of the segment fractions against the adsorption time for SL-PMMA adsorbed from carbon tetrachloride solution. $\triangle$, train; $\square$, short loop; $\bigcirc$, long loop.

In Figure 2, the segment fractions of train, short loop, and long loop for the adsorption of SL-PMMA are plotted as a function of the 
adsorption time. The values of $p$ decrease with the adsorption time, while the fractions of long loop and short loop segments increase. After 4 hours, the values of the segment fractions are almost constant. In the initial state of adsorption, most of the segments were highly localized near the surface. As a result, the adsorbed polymer was considered to be flattened, forming a thin layer. At the saturated adsorption, the value of $p$ reduces to 0.37 , which indicates the adsorbed PMMA has a flat conformation.

In Figure 3, the segment fractions of train, short loop and long loop for the adsorption of SL-PS are plotted as a function of the adsorption time. The values of $p$ decrease with the adsorption time, while the fractions of short loop segments increase. After 4 hours, the values of the segment fractions are almost constant. In contrast to the adsorption of PMMA, the trend toward lower values of $p$ indicates that a more extended adsorbed structure (i.e., loopy conformation) is formed as the silica surface is filled with the adsorbed

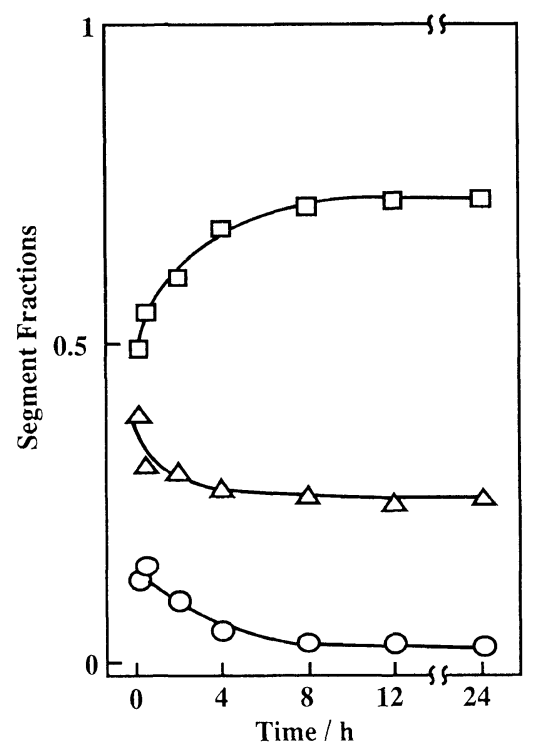

Figure 3. Plots of the segment fractions against the adsorption time for SL-PS adsorbed from carbon tetrachloride solution. $\triangle$, train; $\square$, short loop; $\bigcirc$, long loop.
PS.

\section{Displacement Adsorption of PMMA and PS System}

When PS are adsorbed on silica surface from carbon tetrachloride solution to saturation and then excess PMMA solution of known concentration are added, the amounts of adsorption of PMMA and PS are changed with the adsorption time as shown in Figures 4 and 5. The numbers in the graph are the values of $p$ at that point. The adsorbed amounts of PS represents the desorbed amounts of PS by the adsorbed PMMA. As shown in Figures 4 and 5 , PS is desorbed from silica surface accompanied by an increase in the adsorption time after adding the PMMA solution and finally PS is completely replaced by PMMA. Therefore, this indicates the stronger interaction of PMMA with the silica surface arising from its polar character and greater hydrogen bonding ability. The amount of adsorption for PMMA which saturates the silica surface is obviously lower than that when adsorbed on silica surface

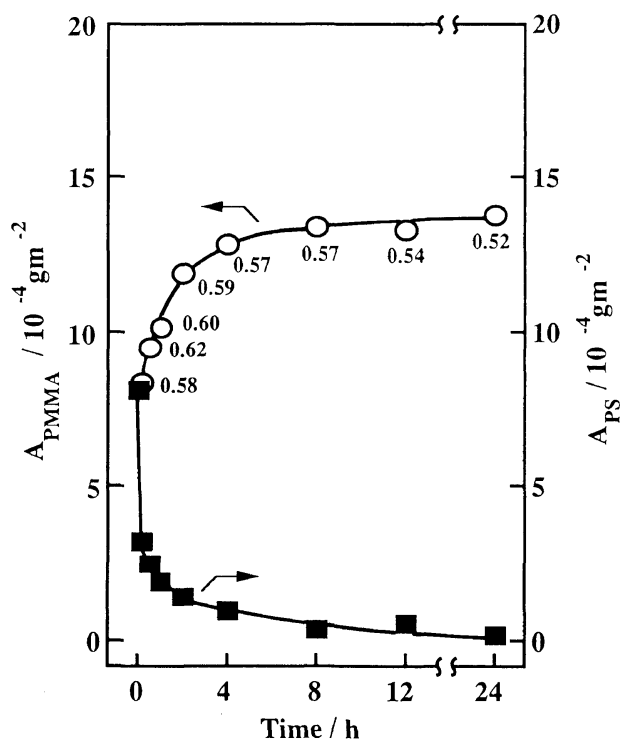

Figure 4. Plots of the adsorbed amounts of SL-PMMA and the desorbed amounts of NL-PS against the adsorption time. The numbers in the graph are the values of $p$ at that point. O, SL-PMMA; $\square$, NL-PS. 


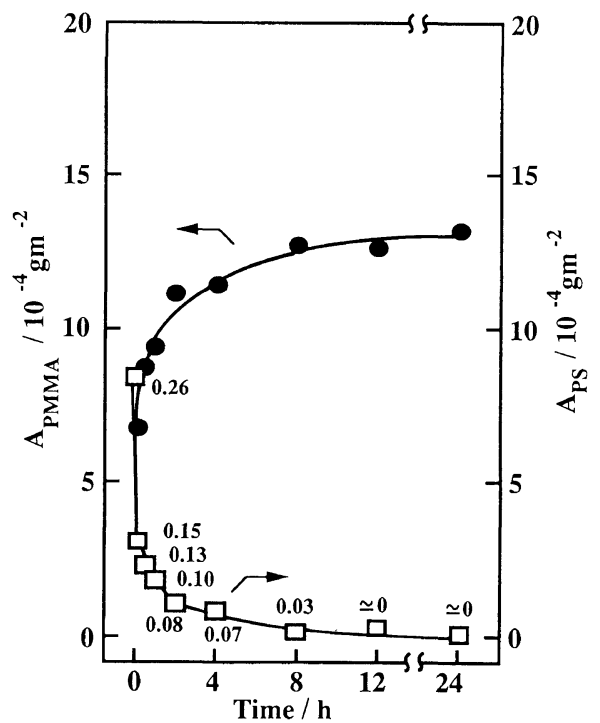

Figure 5. Plots of the adsorbed amounts of NL-PMMA and the desorbed amounts of SL-PS against the adsorption time. The numbers in the graph are the values of $p$ at that point. O, NL-PMMA, $\square$, SL-PS.

individually as shown in Figure 1; that is, accompanied by the displacement of PS, the adsorbed PMMA has a flatter conformation $(p=0.52)$ than that in the individual adsorption $(p=0.37)$. In conclusion, it is clarified that the PS molecule in solution affects the amount and the conformation of the adsorbed PMMA. The results represented here are consistent with our previous observations relating to the competitive adsorption of PMMA and PS mixtures. ${ }^{8}$

In Figure 6, the segment fractions of train, short loop and long loop for SL-PMMA are plotted as a function of adsorption time accompanied by the displacement of NL-PS. As shown in Figure 6, the values of $p$ decrease gradually, while the fractions of long loop segments increase. This adsorption behavior of SL-PMMA is similar to that in the individual adsorption. In the initial state of displacement adsorption, the value of $p$ for SL-PMMA is relatively high ( $p=0.6$; i.e., flat conformation). This reason would be due to the low surface coverage, $\theta_{\mathrm{IR}}\left(\theta_{\mathrm{IR}}=0.16\right)$ of silica surface saturated by $\mathrm{PS}$, as reported previously. ${ }^{6}$

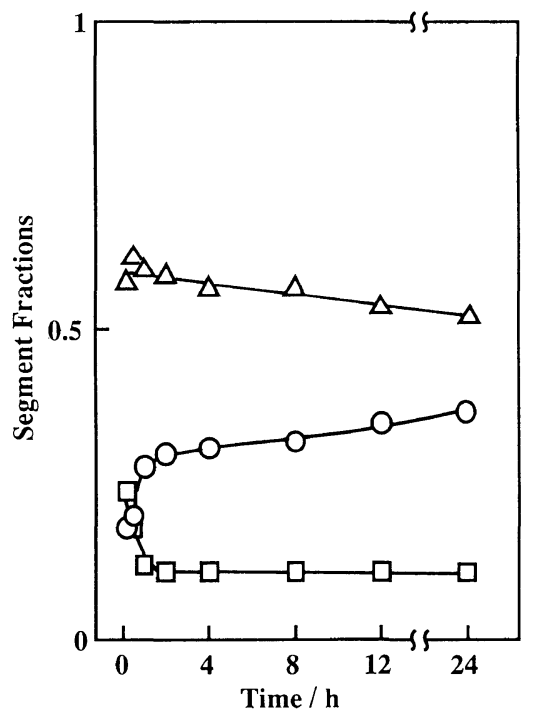

Figure 6. Plots of the segment fractions of SL-PMMA against the adsorption time, accompanied by the displacement of NL-PS. $\triangle$, train; $\square$, short loop; $\bigcirc$, long loop.

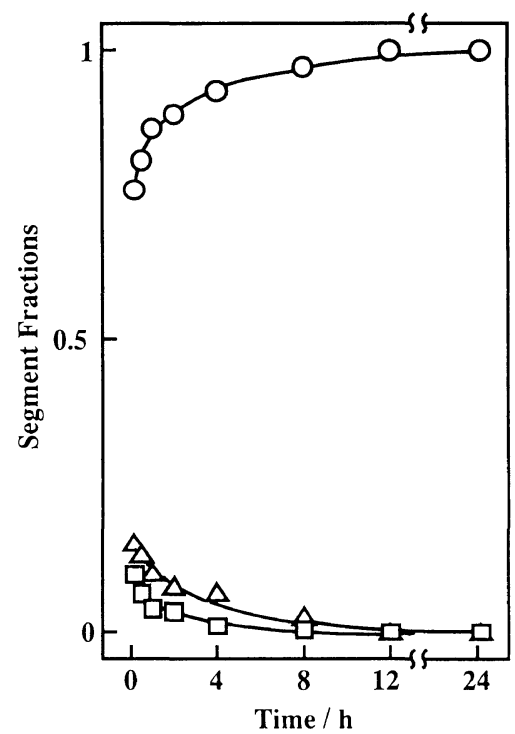

Figure 7. Plots of the segment fractions of SL-PS against the adsorption time, accompanied by the displacement of SL-PS. $\triangle$, train; $\square$, short loop; $\bigcirc$, long loop.

Therefore, PMMA molecules may adsorb to the open space of the surface, having relatively high value of $p$. 
In Figure 7, the segment fractions of train, short loop and long loop for SL-PS are plotted as a function of adsorption time accompanied by the displacement of SL-PS. As shown in Figure 7 , the fractions of train segments and short loop segments decrease dramatically with the adsorption time, while the fraction of long loop segments increases. That is, the adsorbed PS is dramatically deformed into a loopy conformation in the process of desorption.

Comparing the values of $A$ for SL-PMMA in Figure 1 with those in Figure 4 , it is concluded as follows. (1) With the displacement of PS from the silica surface, the amount of adsorption for PMMA which saturates the silica surface is obviously lower than that when adsorbed on silica surface individually; that is, the adsorbed PMMA has a flatter conformation $(p=0.52)$ than that in the individual adsorption ( $p=0.37)$. Therefore, it is clarified that the PS molecule in solution affects the amount and the conformation of the adsorbed PMMA. The results represented here are consistent with our previous observations relating to the competitive adsorption of PMMA and PS mixtures. ${ }^{8}$ (2) With the displacement of PS from the silica surface, it takes a long time for PMMA to adsorb on the silica surface compared with the individual adsorption, because a greater number of PS-silica surface attachments must be ruptured in order to effect displacement.

Significantly, PMMA displaces PS from the silica surface even though PS and PMMA are incompatible polymers. The fact that PMMA completely displaces PS from the silica surface establishes that PMMA readily penetrates the adsorbed PS layer. As soon as the silica surface is saturated with both PMMA and PS, PMMA molecules in solution displace PS molecules.

\section{REFERENCES}

1. H. Sakai, T. Fujimori, and Y. Imamura, Bull. Chem. Soc. Jpn., 53, 3457 (1980).

2. H. Sakai and Y. Imamura, Bull. Chem. Soc. Jpn., 53, 1749 (1980).
3. H. Sakai, T. Asakura, K. Suzuki, K. Horie, Y. Maeshima, and Y. Imamura, Bull. Chem. Soc. Jpn., 54, 2180 (1981).

4. H. Sakai and Y. Imamura, Bull. Chem. Soc. Jpn., 60, 1261 (1987).

5. K. Kobayashi, K. Araki, and Y. Imamura, Bull. Chem. Soc. Jpn., 62, 3421 (1989).

6. K. Kobayashi, H. Yajima, Y. Imamura, and R. Endo, Bull. Chem. Soc. Jpn., 63, 1813 (1990).

7. K. Kobayashi, K. Araki, Y. Imamura, and R. Endo, Bull. Chem. Soc. Jpn., 63, 511 (1990).

8. K. Kobayashi, S. Sugimoto, H. Yajima, K. Araki, Y. Imamura, and R. Endo, Bull. Chem. Soc. Jpn., 63, 2018 (1990).

9. K. G. Barnett, T. Cosgrove, B. Vincent, D. S. Sissons, and M. Cohen-Stuart, Macromolecules, 14, 1018 (1981).

10. T. Cosgrove and K. G. Barnett, J. Magn. Reson., 43, 15 (1981).

11. B. J. Fontana and J. R. Thomas, J. Phys. Chem., 65, 480 (1961).

12. C. Thies, J. Phys. Chem., 70, 3783 (1966).

13. C. V. Linden and R. V. Leemput, J. Colloid Interface Sci., 67, 48 (1978).

14. M. Kawaguchi, K. Hayakawa, and A. Takahashi, Polym. J., 12, 265 (1980).

15. M. Kawaguchi and A. Takahashi, J. Polym. Sci., Polym. Phys. Ed., 18, 2069 (1980).

16. M. Kawaguchi, K. Maeda, T. Kato, and A. Takahashi, Macromolecules, 17, 1666 (1984).

17. M. Kawaguchi, Adv. Colloid Interface Sci., 32, 1 (1990).

18. J. M. Kolthoff and R. G. Gutmacher, J. Phys. Chem., 56, 710 (1952).

19. G. J. Howard and P. McConnell, J. Phys. Chem., 71, 2981 (1967).

20. M. Kawaguchi, M. Chikazawa, and A. Takahashi, Macromolecules, 22, 2195 (1989).

21. M. Kawaguchi, T. Hada, A. Takahashi, Macromolecules, 22, 4045 (1989).

22. A. Takahashi, "Frontiers of Macromolecular Science,” T. Saegusa, T. Higashimura, and A. Abe, Ed., IUPAC, 1989, p 331.

23. M. Kawaguchi, A. Sakai, and A. Takahashi, Macromolecules, 19, 2952 (1986).

24. M. Kawaguchi, T. Itoh, S. Yamagiwa, and A. Takahashi, Macromolecules, 22, 2204 (1989).

25. P. G. de Gennes, "Studies in Polymer Science," Vol. 2, M. Nagasawa, Ed., Elsevier, Amsterdam, 1988, pp 315-331.

26. M. A. Cohen-Stuart, G. J. Fleer, and J. M. H. M. Scheutjens, J. Colloid Interface Sci., 97, 515 (1984).

27. M. A. Cohen-Stuart, G. J. Fleer, and J. M. H. M. Scheutjens, J. Colloid Interface Sci., 97, 526 (1984).

28. M. J. Schick and E. N. Harvey, J. Polym. Sci., B, 7, 495 (1969).

29. S. L. Regen, J. Am. Chem. Soc., 97, 3108 (1975). 